\title{
Femtosecond Laser Structuring in Optical Fiber and Transparent Films
}

\author{
Peter R. Herman, Kyle H. Y. Cheng, Jason R. Grenier, Moez Haque, Kenneth K.C. Lee \\ Department of Electrical and Computer Engineering, University of Toronto, 10 King's College Road, Toronto, Ontario, M5S 3G4, Canada \\ p.herman@utoronto.ca
}

\begin{abstract}
Femtosecond laser processing is optimized for writing optical circuits, optical resonators, and microfluidic devices inside the cladding of single-mode optical fiber that couple efficiently with the fiber core waveguide. The laser processes open new directions towards Labon-a-Fiber.
\end{abstract}

\section{Introduction}

Femtosecond laser direct waveguide writing has shown unique potential in writing three-dimensional (3D) optical circuits [1] in bulk glasses such as fused silica. However such optical circuits typically required connection or coupling into optical fibers. In this paper, we seek to extend femtosecond laser writing techniques into the fusedsilica cladding of conventional single mode optical fibers (SMF) [2,3] and thereby write 3D optical circuits that seamlessly interconnect with the fiber core waveguide. We further harness chemical etching of laser-generated nanogratings to embed microfluidic channels and optical resonator components within the fiber. In this way, femtosecond laser writing facilitates flexible approaches for coupling and integrating multiple optical and microfluidic components to improve the functionality of optical fiber. This approach promises benefits of reduced assembly and packaging costs for a broad range of applications in optical communication, sensing, biomedical catheters and lab-on-a-fiber applications.

\section{Methodology}

A Yb-doped fiber chirped pulsed amplified femtosecond laser (IMRA America; $\mu J$ Jewel D-400-VR) was frequency doubled to $522 \mathrm{~nm}$ and $200 \mathrm{fs}$ pulse duration, and operated at a repetition rate of $500 \mathrm{kHz}$ to drive strong interactions in the fused silica optical fiber [5]. A strongly focusing (100X,1.25NA) oil-immersion lens was used to provide tight confinement and strong refractive index contrast while also avoiding beam distortion by spherical aberration at the fused silica fiber surface. An acousto-optic modulator provided laser burst trains for writing of segmented waveguides with Bragg resonances in the Telecom band. Optical circuits were written into standard single-mode fiber (SMF-28) and further tested in pure fused silica fiber of similar diameter $(125 \mu \mathrm{m})$. Fusion splicing of the laser-modified coreless fiber to SMF was studied as an alternate opto-fluidics fiber platform.

Optical characterization of the laser-structured devices was made by probing with fiber coupling according to the procedures previously described in [2]. All optical spectra were recorded with an optical spectrum analyzer and normalized against fiber-to-fiber coupling with index matching oil applied at all optical interfaces to reduce Fresnel reflection loss. Free-space lens firing into the end facet of waveguides was used for characterization of the waveguide and device birefringence.

\section{Results and Discussion}

Various designs of waveguide couplers, including directional (Fig. 1a), S-bend (Fig. 1b) and cross (Fig. 1c) were first written in bulk fused silica glass to study the coupling responses and establish the approximate laser processing windows and coupler designs for introduction into both the SMF and coreless fiber. The spectral bandwidth and coupling efficiencies of each of the coupler types were examined towards applications in creating power taps, multiwaveguide splitters, Bragg-grating strain gauges and interferometric filters. A Mach-Zehnder Interferometer (MZI) was laser written in coreless fused silica fiber to demonstrate a new direction for fabricating compact wavelength division multiplexing (WDM) devices.

An example of a cross coupler of the design in Fig. 1c is shown in the optical image of Fig. 1d for the case of coreless waveguide. The main arm of the cross coupler was first fabricated with $130 \mathrm{~nJ}$ pulse energy at $0.25 \mathrm{~mm} / \mathrm{s}$ writing speed. The split arm was written at similar laser exposure over a wide range of angles $\theta$ followed by a curved section of $35 \mathrm{~mm}$ radius of curvature $(R)$ to bring the spur waveguide parallel with the center waveguide. Figure 2a) shows the relative coupling ratio measured into each arm versus angle $\theta$ at $1550 \mathrm{~nm}$, that follows the

This is an Open Access article distributed under the terms of the Creative Commons Attribution License 2.0, which permits unrestricted use, distribution, and reproduction in any medium, provided the original work is properly cited. 
expected theoretical trends for such a device [4]. Optimal 50/50 coupling was observed around $\theta=2.5^{\circ}$. The combined loss of both arms amounts to $\sim 3.6 \mathrm{~dB}$ (not shown in Fig. 2a)). Similar coupling ratio results were also observed in writing S-bend couplers in SMF fiber, with the added benefit of lower propagation loss in the SMF core waveguide. S-bend waveguides were also found to offer a reproducible and controllable coupling to the laser formed spur-waveguide in both SMF and coreless fiber. A key requirement for reproducibility was the precise centering of the laser focus to the center or core waveguide in the fiber. By following the back-reflected laser beam from the fiber cladding surfaces, laser alignment techniques were developed that offered $\pm 1 \mu \mathrm{m}$ centering precision.

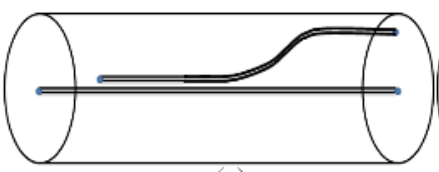

(a)

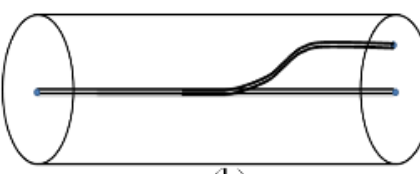

(b)

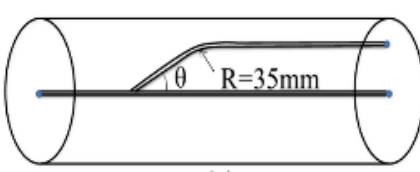

(c)

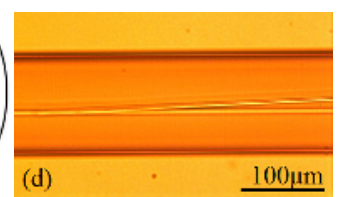

Fig. 1. Coupler designs tested in SMF and coreless fiber: (a) directional, (b) S-bend, (c) cross and (d) image of cross coupler in coreless fiber.

A design for a one to four splitter consisting of two S-bends $(\mathrm{R}=35 \mathrm{~mm})$ directed diagonally upward $\left( \pm 45^{\circ}\right.$ azimuthal) and two cross couplers written downward $\left( \pm 135^{\circ}\right.$ azimuthal) is shown schematically in Fig. $2 b$ (inset). This splitter has nearly even splitting on all four arms across a very wide spectral band of 1250 to $1700 \mathrm{~nm}$ as shown in the transmission spectra in Fig. 2b. The arms of a similar 3:1 splitter were formed into a distributed optical shape and temperature sensor, with three Bragg grating waveguides formed along three different waveguide arms. This grating configuration offered unambiguous 3D shape and temperature sensing.
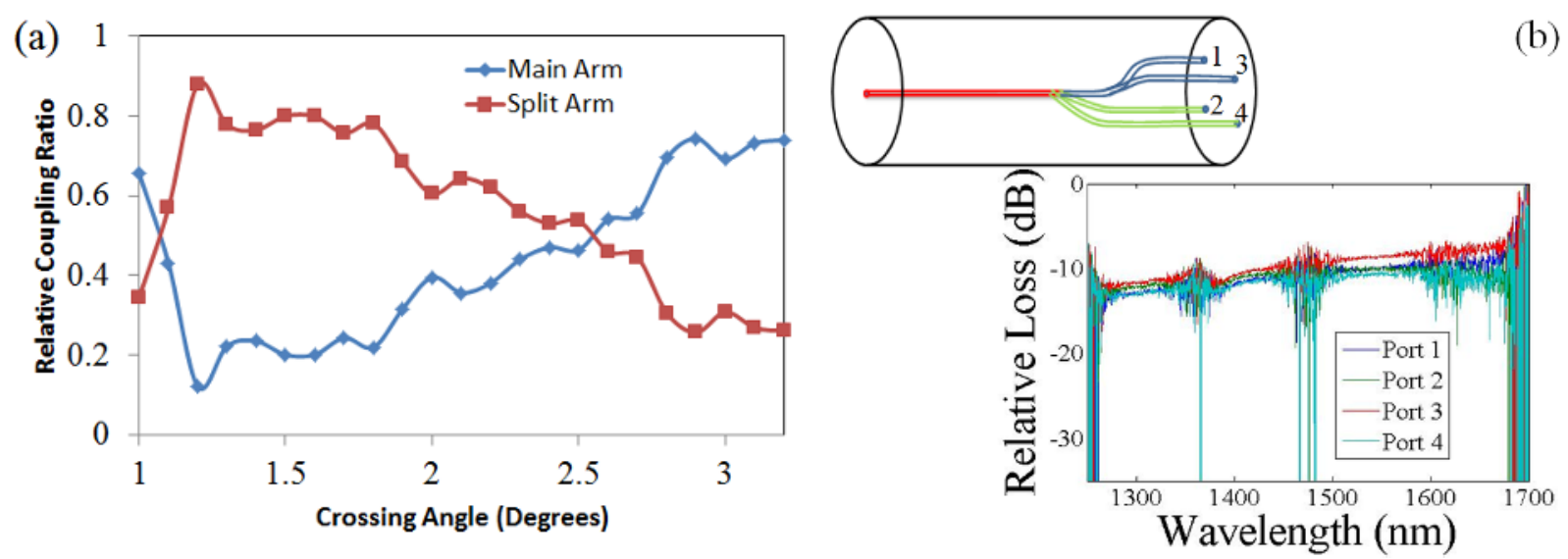

Fig. 2. (a) Measured optical coupling ratio into the main and spur arm waveguides in a laser writing X-couplers versus the crossing angle for coreless fiber. (b) A hybrid S-bend and X-type coupler with one-to-four waveguide splitting depicted in the inset image together with the nearly equally balanced transimission spectrum measured from each port across the Telecom Bands.

The study of directional couplers showed a much wider range of spectral properties, particularly a strong wavelength dependence that would be attractive for WDM application. Overall coupling was less efficient in SMF than in coreless figer due to beta-mismatch between the laser-formed waveguide and the doped-core of the SMF fiber. A comparison of the types of couplers studied in SMF and coreless fiber had the following overall properties:

- Insertion losses were lowest in the SFM due to the low propagation loss of the waveguide core and the efficienct optical coupling with the fiber-based optical characterization system.

- Laser formed waveguides in coreless fiber were found to erase during fusion splicing with SMF to lengths of several hundred microns from the splice point. Hence, waveguides were formed in coreless fiber after the fusion splicing step. In this approach, the laser-formed waveguides could be aligned to the SMF core waveguide and yield a typically $3 \mathrm{~dB}$ insertion loss at each splicing point.

- S-bend and X-couplers were developed to offer a wide range of controllable coupling ratio $(<1 \%$ to $\sim 80 \%)$ from the core SMF waveguide to the laser-formed spur waveguide, with highly uniform response across the 1250 to $1700 \mathrm{~nm}$ spectrum studied here.

- Directional couplers yielded a wide range of coupling efficiency with a strong wavelength dependence that could be enhanced or diminshed by the coupler design (waveguide-to-waveguide separation). The spectral properties of directional couplers formed in coreless fiber were more reproducible than the couplers formed 
in SMF due to the uncertain $\pm 1 \mu \mathrm{m}$ positioning of the laser-formed waveguide relative the pre-existing core waveguide. A comprehensive reporting of the coupler characteristics will be reported in a future publication.

A MZI was designed for the coreless silica fiber as shown in Fig. 3a. The main arm of the MZI was fabricated at the center of the fiber with $110 \mathrm{~nJ}$ pulses while the side arm was fabricated with $8 \mathrm{~S}$-bends with stronger $230 \mathrm{~nJ}$ pulse energy exposure to give a bigger optical path difference. The resulting transmission spectrum is shown in Fig. $3 \mathrm{~b}$ to have a free spectral range of $55 \mathrm{~nm}$.

(a)
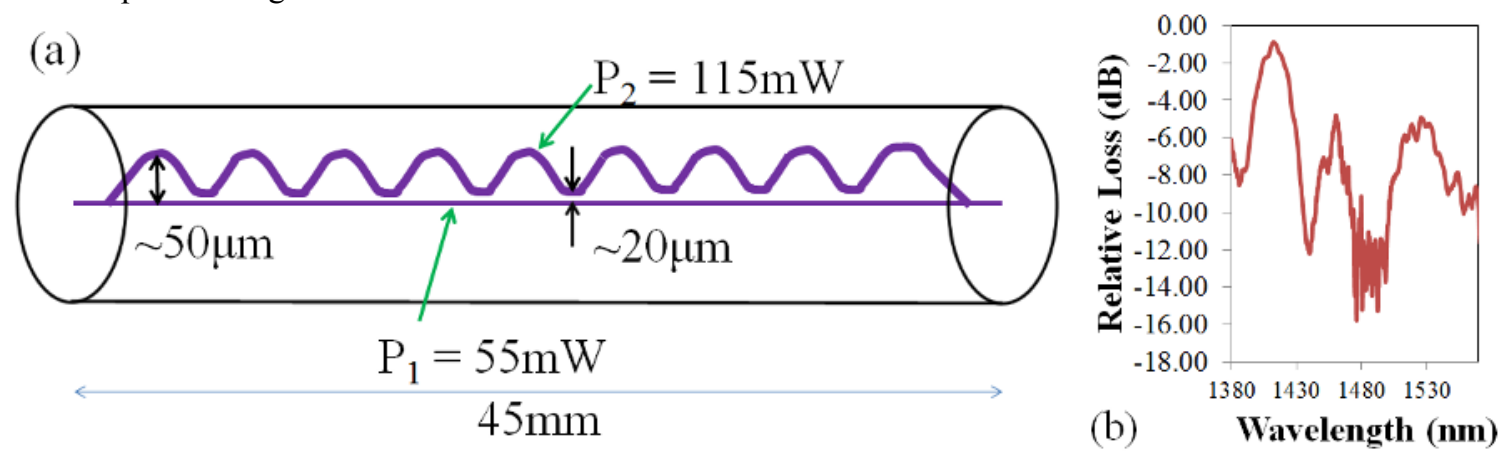

Fig. 3. (a) Design of a femtosecond laser written MZI in coreless silica fiber and (b) the recorded transmission spectrum.

The fiber-integrated MZI demonstrates the potential for tailoring the spectrum and introducing WDM devices into optical fiber by laser writing through the fiber side. This approach opens a unique opportunity for integrated devices in fiber, allowing application specific wavelength filtering or wavelength division multiplexing applications in communication or sensing application with advantages in low packaging and assembly cost.

A LIF concept demonstrated here in Fig. 4 is the formation of a 3D microfluidic network embedded within coreless fiber. Laser exposed coreless and SMF fiber was chemically etched in 5\% HF acid for 2 hours to open laser tracks. This representative example splits a single microchannel into four separate radial arms that are independently probed by laser-formed waveguides (not shown). Very smooth cavity walls of $12 \mathrm{~nm}$ (rms) roughness were measured by AFM. With this procedure, a Fabry-Perot cavity was formed in coreless fiber and probed with laser-formed waveguides to define a novel sensor platform. More elaborate 3D optofluidic networks may be envisioned over long lengths of optical fiber to further exploit the benefits of undistorted laser writing with the oil immersion lens and thereby interconnect optical waveguides with microchannels in straight, circular, or arbitrarily complex shapes.
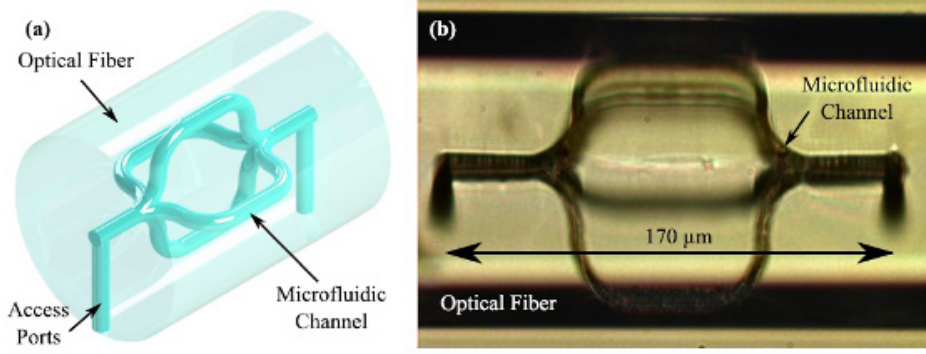

Fig. 4. (a) Schematic and (b) optical microscope image of a 3D microfluidic network embedded within optical fiber of $125 \mu \mathrm{m}$ diameter. A single microchannel is split into four separate radial arms that may each be independently probed by laser-formed waveguides (not shown). The channels opened along laser exposure tracks formed with oil immersion focusing to avoid optical aberrations. This undistorted patterning of nanograting structures offered precise formation of straight and circular channels after HF etching.

\section{References}

[1] Catalin Florea and Kim A. Winick, "Fabrication and characterization of photonic devices directly written in glass using femtosecond laser pulses," J. Lightwave Technol. 21, 246-253 (2003).

[2] H. Zhang, S. M. Eaton, and P. R. Herman, "Single-step writing of Bragg grating waveguides in fused silica with an externally modulated femtosecond fiber laser," Opt. Lett. 32, 2559-2561 (2007).

[3] J. R. Grenier, L. A. Fernandes, P. V. Marques, J. S. Aitchison, and P. R. Herman, "Optical Circuits in Fiber Cladding: Femtosecond laser-written Bragg grating Waveguides," in CLEO:2011 - Laser Applications to Photonic Applications, OSA Technical Digest (CD) (Optical Society of America, 2011), paper CMZ1. [4] Florian Dürr and Hagen Renner, "Analytical Design of X-Couplers," J. Lightwave Technol. 23, 876- (2005).

[5] Lawrence Shah, Alan Arai, Shane Eaton, and Peter Herman, "Waveguide writing in fused silica with a femtosecond fiber laser at $522 \mathrm{~nm}$ and $1 \mathrm{MHz}$ repetition rate," Opt. Express 13, 1999-2006 (2005). 